\title{
En busca de la Tecnología Educativa: la disrupción desde los márgenes
}

\section{Searching for Educational Technology: disruption from the edges}

\author{
$M^{a}$ Paz Prendes Espinosa \\ Universidad de Murcia \\ pazprend@um.es \\ José Luis Serrano Sánchez \\ Universidad de Murcia \\ jl.serranosanchez@um.es
}

Recibido: $25 / 06 / 2016$

Aceptado: $30 / 06 / 2016$

Publicado: 30/06/2016

\section{RESUMEN}

Este artículo es la presentación de la nueva revista científica sobre investigación en Tecnología Educativa (RIITE) en su número 0 inaugural. Pretendemos explicar de dónde y por qué surge la revista y, de modo paralelo, justificar la importancia de una publicación orientada a la difusión científica de los trabajos de investigadores noveles. Además de ello analizamos algunos aspectos de la revolución que las tecnologías digitales han promovido en este ámbito de la difusión científica y la publicación de revistas: plataformas como OJS, por una parte, pero también las métricas de impacto o las publicaciones digitales de acceso abierto.

Creemos que dar voz a los investigadores noveles es no solamente una idea original, sino además es una necesidad de vital importancia por las dificultades que cada vez más a menudo encuentran, dado el cariz que han ido tomando las revistas que de forma generalizada se han visto sometidas a los criterios de indexación y de impacto.

Por último, la disrupción desde los márgenes supone enfocar la investigación en Tecnología Educativa asumiendo el valor no solamente de la comunidad de investigadores en formación para presentar sus trabajos sino para contribuir a la difusión y creación de conocimiento a través de las redes sociales y herramientas de gestión de la información. Así pues, nuestra comunidad y con ella la evolución de las redes telemáticas serán el sustento que irán marcando la evolución de nuestros sistemas de difusión.

\section{PALABRAS CLAVE}

Tecnología educativa; investigación; acceso abierto; impacto.

\section{ABSTRACT}

This article is our presentation of the new scientific review about Educational Technology Research (RIITE) in this first number. Our intention is to explain the birth of this review - why and how- and also to justify the relevance of novel's research and its diffusion. Moreover we analyze some aspects of digital revolution relative to scientific diffusion and periodical reviews; we are going to mention: OJS environment, impact metrics or open access.

We believe that novel researchers must have voice and this is really an original idea because of the way that traditional reviews have been going these last years. Indization and impact are really conditioning publication and it is more and more difficult to publish if you are not a consolidated researcher with a founded project.

Last but not least, disruption from the edges means to focus Educational Technology research assuming not only the value of research done by novel researchers but also their contribution to knowledge using social nets and different telematic tools to knowledge management. So our community and the evolution of telematic tools will be the keys to write our way.

KEYWORDS (arial 10, negrita)

Educational technology; research; open access; impact.

\section{CITA RECOMENDADA}

Prendes, M.P. y Serrano, J.L. (2016). En busca de la Tecnología Educativa: la disrupción desde los márgenes. RIITE. Revista Interuniversitaria de Investigación en Tecnología Educativa, 0, 6-16. Doi: http://dx.doi.org/10.6018/riite/2016/263771 


\section{BIENVENIDOS AL NACIMIENTO DE RIITE}

Nace una nueva revista y con ella muchas ilusiones y también mucho trabajo. En los tiempos actuales, en los que la digitalización y las redes han supuesto -al igual que en otros ámbitos- un impresionante cambio en el mundo de la difusión científica, puede sorprender que queramos lanzar otra revista, otra más de entre las muchas que ya hay. Veamos en esta presentación los motivos que nos hacen iniciar este proyecto con la ilusión de que pueda consolidarse como un espacio útil y de interés para la difusión científica.

La revista surge en el contexto del consorcio de universidades (Rovira i Virgili, Lérida, Islas Baleares y Murcia) responsables del programa de "Doctorado Interuniversitario en Tecnología Educativa" y del "Máster Interuniversitario en Tecnología Educativa: E-Learning y Gestión del Conocimiento". Todo el equipo docente de estos programas formativos participará en las tareas de evaluación propias de la revista, siendo el equipo de la Universidad de Murcia (el Grupo de Investigación en Tecnología Educativa) quien asumirá el trabajo de edición y gestión con la colaboración de Editum, Servicio de Publicaciones de la Universidad de Murcia.

La Revista Interuniversitaria de Investigación en Tecnología Educativa (RIITE) surge con la finalidad de promover y compartir los avances en investigación en el área de la Tecnología Educativa. Nuestra finalidad es la difusión de investigaciones originales en sus diversas fases del proceso, proporcionando visibilidad a diversos proyectos, tanto financiados como no financiados. Nos interesa publicar trabajos que no solamente describan los aspectos más relevantes de la investigación de forma breve, sino que contengan enlaces a los instrumentos de recogida de datos, enlaces a bases de datos reutilizables o enlaces a recursos de investigación que puedan ser útiles a otros investigadores.

Esta propuesta editorial tiene especial interés en contribuir a la difusión de investigaciones realizadas por investigadores en formación (noveles), habitualmente estudiantes de másteres y de doctorados que encuentran numerosas dificultades para publicar cuando sus investigaciones no están ligadas a proyectos financiados de otros investigadores consolidados. A esta circunstancia tenemos que añadirle el factor tiempo, pues por experiencia personal y repetida sabemos que un autor puede ver pasar más de un año hasta obtener una respuesta por parte de una revista. En caso de que el artículo haya sido aceptado, la espera se da por buena; en caso contrario, suele ocurrir que el artículo ya no sirve, pues su información ha quedado desfasada y obsoleta, más aún en un ámbito de tan exigente actualización como es el de la Tecnología Educativa.

Estas dificultades de publicación a las que estamos aludiendo son aún mayores si el autor del artículo es un estudiante de máster o doctorado que, en la mayoría de los casos, está comenzando su camino con una investigación local, de escaso alcance, sin financiación y sin un proyecto detrás que lo sustente. Es por ello que precisamente son los investigadores noveles y en formación quienes más interés pueden tener en la existencia de espacios de publicación como éste que inauguramos y que espera contribuir de forma eficaz a integrarlos en las redes de información de los investigadores científicos y a promover la difusión de sus trabajos. Todos hemos tenido nuestra primera investigación, siempre hay un comienzo y siempre es complicado. Esta revista viene a intentar cubrir ese espacio y esta idea, original tanto desde una perspectiva nacional como internacional, es disruptiva porque viene a romper con las tendencias que sin duda se están consolidando como tradición en el ámbito de la investigación científica y que dificultan, sin duda, la difusión de estos pequeños trabajos que no carecen de valor. Así pues, estos investigadores noveles que a menudo se sienten en el margen del sistema, podrán encontrar en nuestra revista un espacio de publicación de sus trabajos -previa evaluación ciega-.

Otro elemento de interés en relación con este tema de las publicaciones científicas es el del impacto. En los últimos años hemos podido ver cómo han ido surgiendo numerosas revistas nuevas, pues cada vez los investigadores somos más conscientes de la importancia de la difusión científica, pero también somos más conscientes, no nos engañemos, de la necesidad

\footnotetext{
${ }^{1}$ Web del Doctorado de Tecnología Educativa: http://gte.uib.es/doctorado-te/

${ }^{2}$ Web del Máster de Tecnología Educativa: http://www.tecnologiaeducativa.eu/
} 
de publicar para poder superar nuestras evaluaciones, tramos, sexenios, acreditaciones y demás procesos de reconocimiento de nuestro trabajo. A la par, hemos ido introduciendo en nuestro ámbito educativo los conceptos de "impacto" y "relevancia" que años atrás no sabíamos ni cuál era su significado.

Coincidimos con Ruiz-Cobella, Galán y Diestro (2014) en que el notable incremento de la creación de revistas científicas comenzó tras los cambios del modelo de evaluación de la carrera docente-investigadora del profesorado de cara a su selección y promoción. La aprobación de la Ley de Reforma Universitaria en 1983, el Real Decreto 1086/1989 de 28 de agosto, la Ley Orgánica de Universidades de 2001 y su posterior modificación en 2007 han modificado sustancialmente los mecanismos de publicación de los investigadores. De manera paulatina se otorgó más valor a los artículos científicos como evidencias de una actividad investigadora de calidad, en detrimento del que hasta el momento había sido el principal canal de comunicación elegido por los investigadores: el libro. Según los autores, el aumento del número de Facultades de Ciencias de la Educación y Escuelas Universitarias de Magisterio y la organización de la universidad española en departamentos y áreas de conocimiento, ha contribuido también a la proliferación de revistas en Ciencias de la Educación en las últimas tres décadas. En estos casos los objetivos han sido varios: como seña de identidad, como mejora de la visibilidad y/o como búsqueda de reconocimiento entre las distintas comunidades científicas.

\section{EN ABIERTO}

Si el crecimiento de revistas en las tres últimas décadas es evidente, casi más lo es el aumento de revistas electrónicas y de acceso abierto, sumándose RIITE a esta tendencia. Coincidiendo con la llegada de la World Wide Web (WWW), nacieron las primeras revistas electrónicas en 1990, aunque el despegue real se produjo durante la segunda mitad de esta década, pasando de quinientas revistas en 1996 a más de cuatro mil en el año 2000. Sus incuestionables ventajas son el principal motivo de este espectacular crecimiento (Delgado, 2015):

- Rapidez en el sistema de producción editorial.

- Capacidad de almacenamiento ilimitado.

- Hipertextualidad.

- $\quad$ Utilización de medios audiovisuales complementarios al artículo.

- $\quad$ Difusión ilimitada.

- Simultaneidad en la consulta.

- Control en el uso y evaluación del impacto.

- Reducción significativa de costes de edición.

- Flexibilidad en la consulta.

- Interactividad entre todos los agentes (autores, revisores, editores y lectores).

A pesar de las ventajas vistas anteriormente sigue sorprendiendo que casi "la mitad de las revistas científicas circulantes en el mundo se sigan editando en papel, porcentaje que se eleva casi al $99 \%$ en las que figuran indizadas en los JCR. Pareciera que el formato impreso todavía se asocia con la reputación y solvencia científica de una revista" (Delgado, 2015, pp. 6-7).

Decíamos que RIITE es una revista electrónica y de acceso abierto. Nos parece básico que las publicaciones científicas estén en abierto para contribuir a la difusión de la información y el conocimiento y el desarrollo de las redes de investigación. Creemos que las publicaciones en línea de acceso abierto son el presente y el futuro de la investigación científica y que posibilitan además una mayor inmediatez en la difusión de la información y la transferencia del conocimiento. Tendencia (revistas electrónicas y de acceso abierto) que ha sido adoptada especialmente durante la última década, aunque sigue siendo una alternativa minoritaria ya que son poco más del $10 \%$ del total de las revistas circulantes. En palabras de Delgado (2015, p.8), parece que "la imagen que se proyecta de las revistas en acceso abierto es la de un mundo alternativo, en los márgenes del núcleo dominante de la comunicación científica. Es justamente por eso por lo que genera todavía recelos y dudas sobre su credibilidad científica". 
Otro aspecto, más allá de la credibilidad científica de las revistas de acceso abierto, es el modelo de negocio que durante muchos años ha figurado -y figura- en la publicación de artículos. Desde el movimiento de acceso abierto (véase Arnaiz y Prendes, 2010) los investigadores tienen la posibilidad de utilizar y desarrollar sus propios medios de comunicación, con lo que la intermediación que ofrecían las revistas podrían entrar en crisis y disminuir el número de suscripciones. La relación entre ciencia y economía es obvia e incluso necesaria. Durat y Mengual-Andrés (2014, p.5) afirman que hay una "vinculación existente entre la productividad científica de un país (medida a través de su presencia en el JCR) y el crecimiento o desaceleración de su PIB".

Sin embargo, no compartimos la reacción de muchas editoriales a la situación de cambio que propone el movimiento de acceso abierto, la cual ha consistido en la modificación de redirigir los ingresos de un sitio a otro, es decir, cobrar por publicar en vez de por leer (Aliaga, 2014). De esta manera se autodenominan revistas de acceso abierto y además hacen sostenible el modelo de negocio, lo cual les permite contar con mayores recursos para figurar en las más prestigiosas bases de datos, que recordemos suelen tener a su vez fines comerciales. Tampoco compartimos la irresponsabilidad social de investigadores que con fondos públicos deciden publicar en revistas que dificultan el acceso a los contenidos por mantener el modelo convencional de publicación académica sostenido por editoriales comerciales. Sin duda, las políticas de acceso abierto que muchos gobiernos han adoptado en los últimos años podrán desterrar este tipo de prácticas, exigiendo que los resultados de las investigaciones financiadas con fondos públicos sean accesibles al público (Weller, 2014).

La política de RIITE al respecto es clara: para los autores no existen cargos -ni de envíos ni de publicación- y de igual modo tampoco los hay para los lectores. El equipo editorial asume las posibles limitaciones que esto pueda ocasionar para el crecimiento de la revista al contar con menos recursos, pero mantenemos y respetamos la filosofía que subyace al movimiento de acceso abierto. Es por tanto una revista comprometida con una ciencia que contribuya al bienestar social público de forma responsable, alejada de comercializar el conocimiento. Es un proyecto científico que no tiene como única meta ser incluido en listados, y no porque vayamos a despreciar la calidad de la misma, sino porque sigue existiendo controversia en relación a los sistemas de medición de la calidad de los trabajos que utilizan índices como JCR o SJR. En estos casos utilizan realmente el factor de impacto como herramienta de comparación de revistas, y no como sistemas de medición eficaces de la calidad de los trabajos de investigación (Durat y Mengual-Andrés, 2014).

\section{LA DISRUPCIÓN DESDE LOS MÁRGENES}

Como consecuencia de las posibilidades de las tecnologías y los cambios culturales provocados durante las últimas décadas con la llegada de Internet, el movimiento de acceso abierto a la información y al conocimiento ha generado y genera tensiones por el terremoto que ha provocado en el sistema más tradicional de publicación académica y en los intereses por las editoriales comerciales en mantener su status quo. Además de la publicación en abierto de las revistas científicas, otras formas de comunicación apoyadas en la filosofía open se hacen hueco en los procesos de divulgación del conocimiento, e incluso durante todo el proceso de investigación. Hablamos de aquellas herramientas y estrategias utilizadas que permiten a los investigadores (y docentes también) compartir, reflexionar y colaborar con otros profesionales en entornos virtuales. Digital Scholarship es tal vez el concepto que mejor describe este tipo de prácticas y que desde RIITE tenemos en cuenta para tratar de "estar" en los espacios en los que se crean estas dinámicas.

Este concepto lo entendemos como el conjunto de prácticas que desde el mundo digital desempeñan y reflejan de manera abierta y conectada los investigadores/docentes con otras personas, demostrando así su especialización en su campo (Weller, 2011). Esto puede suponer cambios sustanciales tanto en la enseñanza como en la investigación, habiendo sido objeto de estudio en diversos trabajos (Ren, 2013; Scalon, 2014; Veletsianos 2012; Veletsianos y Kimmons, 2012; Weller, 2011). Entre otros aspectos de interés, en estos trabajos se analiza el interés de las consecuencias de la ruptura con los métodos tradicionales de investigar, los retos y conflictos generados y en la participación Web de los investigadores con la consecuente 
generación de nuevos entornos digitales (Costa, 2013). Esto viene a demostrar que cada más los investigadores "habitan" los medios sociales, compartiendo estos espacios con gran parte del resto de la sociedad. Por este motivo, desde RIITE pretendemos "estar" en estos entornos con el objetivo de acercar el conocimiento al público que pueda estar interesado y lograr un mayor impacto en realidades educativas. Coincidimos con Ruiz-Corbella et al. (2014, p. 18) en su planteamiento del "branding editorial en redes sociales" y es uno de nuestros objetivos llevarlo a cabo con nuestra revista. Usando sus palabras, diremos que:

"cualquier revista científica que se precie, que quiera destacar en el ámbito de la educación en este siglo XXI y conseguir una buena difusión en el medio social, debe conjugar criterios de calidad editorial con el impacto y la gestión 2.0. (...) No solo basta con tener presencia en Google o integrar la revista en OJS, sino que se debe potenciar ese branding editorial en las redes sociales (Twitter, Facebook, Mendeley, etc.) y en las redes propias para investigadores creadas en los últimos años (ResearchGate, Academia.Edu, etc.)".

Los indicadores altmetrics han surgido para tratar de medir el impacto del medio social en la difusión de la producción científica. Desde esta perspectiva se entiende por impacto no solo el número de citas, sino otros aspectos como las visualizaciones del artículo, descargas, menciones en medios sociales, etc. De esta manera, esta nueva "bibliometría es capaz de captar tanto el impacto científico, educativo, profesional como el mediático" (Orduña, Martín y Delgado, 2016, p. 488). Sin duda es una posible alternativa al clásico factor de impacto usado para las revistas científicas y que probablemente vaya superando las limitaciones actuales, debidas en gran parte a su relativa juventud y a la falta de uso generalizado por parte de las revistas científicas. En el estudio realizado por Segado-Roj (2013) sobre el interés de las revistas científicas españolas del área de la comunicación en redes sociales (Facebook y Twitter), se concluye que existe un evidente desinterés generalizado, solo 3 de cada diez revistas utilizan redes sociales para aumentar la visibilidad de los resultados de investigación que publican. Insistimos en que el estudio se centró en revistas del área de la comunicación. Por lo tanto, parece que esta "nueva corriente convivirá todavía con la evaluación basada en revistas, que terminará irremediablemente diluyéndose como un azucarillo" (Orduña, et al., 2016, p. 495).

En otro estudio (Esteban-Vázquez, 2013) se comprobó cuáles son los formatos digitales de divulgación (formato HTML, PDF, EPUB, Redes sociales, video-artículo, foros, app móvil y RSS) y las posibilidades interactivas que ofrecen las páginas Web de las revistas indexadas en JCR del campo de la Comunicación mediante una rúbrica de análisis. El autor propone "un nuevo modelo de divulgación científica que comparta el soporte escrito con el videoartículo, la divulgación en redes sociales y la difusión en formatos soportados por dispositivos digitales móviles que favorezcan una mayor visibilidad internacional del avance científico y social de manera más integrada en la sociedad interconectada y visual en la que vivimos" (EstebanVázquez, 2013, p. 83). Modelo con el que nos sentimos identificados desde RIITE y el cual trataremos de implantar de manera realista y progresiva.

En definitiva, parafraseando a Moreno Olmedilla en el SEP 2016 celebrado en Madrid, en educación la disrupción puede llegar de forma efectiva desde los márgenes del sistema; y es esta idea la que recoge el subtítulo de este artículo por entender que la filosofía que inspira y guiará este proyecto encaja en tal planteamiento. En este caso, entendemos como márgenes del sistema justamente a aquellos que desean formar parte de la estructura pero que encuentran muchas dificultades, a ellos hemos aludido al comienzo: los investigadores noveles. $Y$ entendemos como estar en el margen del sistema el hecho en sí mismo de no buscar el impacto, sino la calidad entendida desde la evaluación científica rigurosa de los textos, sin otra preocupación más que la de querer contribuir al desarrollo del conocimiento científico con la difusión de investigaciones de interés.

\section{INTERESADOS EN LA TECNOLOGÍA EDUCATIVA}

La revista tiene un tema central a partir del cual se juzgará como primer parámetro la idoneidad de las publicaciones y este tema que centra nuestro interés es la investigación en 
Tecnología Educativa. Este concepto, que para algunos puede parecer muy claro, probablemente no resulte tanto si analizamos con cierta perspectiva la evolución reciente de la Tecnología Educativa en España como ámbito de conocimiento dentro del campo de la investigación educativa. Por ejemplo, es frecuente confundir el uso de tecnologías como herramientas de carácter transversal y de propósito múltiple con el uso educativo de tecnologías y la investigación sobre ello. Este tipo de confusiones forma parte de las muchas controversias que se han producido históricamente en este ámbito de conocimiento (Serrano, Gutiérrez y Prendes, 2016).

En relación con la situación actual de la Tecnología Educativa, a modo de metáforas nos vamos a permitir presentar algunas leyes que pueden ser reinterpretadas al albur de la realidad de nuestra área de conocimiento, permitiéndonos a su vez mostrar lo que entendemos por Tecnología Educativa.

\section{- Ley de la Polka}

Es muy habitual encontrar experiencias e investigaciones que de forma repentina descubren que el vídeo sirve para enseñar, o que los alumnos aprenden más allá de lo que los profesores enseñan, o que la personalización de la enseñanza es interesante desde la perspectiva de la heterogeneidad del alumnado. Y así se introducen nuevas siglas y conceptos (el MOOC, el PLE, el aprendizaje adaptativo, etc.) que vienen a redundar en viejas ideas con un gran problema de base: olvidan precisamente que su sustento es viejo. Creen partir de la nada, cuando la tradición de investigación es amplia, diversa y anterior en el tiempo. Nos sirve como ejemplo de ello un informe sobre el uso del vídeo como recurso educativo de Kaltura (2015) en el cual aparecen numerosas ideas propias de la abundante investigación sobre el uso de los medios audiovisuales en los años 80 y 90.

\section{- Ley del bálsamo de Fierabrás.}

No hay una solución mágica ni las tecnologías van a ser la solución de todos los problemas de la educación. Esta idea es un mantra que venimos repitiendo y oyendo desde los mismos albores de la Tecnología Educativa. No obstante, seguimos encontrando trabajos de investigación que parecen haber descubierto esa piedra filosofal que todo lo convertirá en oro. O usando una idea que se atribuye a Chadwick, nos dan un martillo y ya queremos darle a todo un martillazo. O cómo de repente aparecen los MOOC, que desde la pedagogía son considerados en su origen con cierto desprecio por recordarnos viejos tiempos de enseñanza basada en máquinas de enseñar (Skinner) o centrada en contenidos, pero muy al contrario, desde las instituciones y organizaciones educativas parecen haber encontrado la solución a todos los problemas del sistema de enseñanza superior (véase Prendes y Sánchez, 2014).

\section{- Ley del País de las Maravillas}

Las tecnologías tienen, entre otros efectos, uno ciertamente peligroso: su brillo nos deslumbra. Y si nos dejamos deslumbrar por las tecnologías, caeremos en la ley anteriormente explicada y podremos llegar a pensar que todas las soluciones -y la única solución- están en el uso de tecnologías. Asociado a esta idea aparece el gran peligro de pensar que nuestra preocupación deben ser las tecnologías en sí mismas, como meta y como elemento clave de la innovación educativa. Contrariamente a ello, creemos que una perspectiva pedagógica coherente debe proporcionar un sustento sólido a cualquier proceso de innovación educativa apoyado en tecnología. Y a partir de esta idea, lo relevante no lo encontraremos en la técnica o el aparato, sino en las metodologías, en las competencias, en la formación del profesorado, en las necesidades y características del alumnado, en los elementos del contexto... En definitiva, lo educativo sustentando lo tecnológico. No nos dejemos cegar y seamos capaces de configurar un espacio de trabajo sustentando en la investigación y en el conocimiento científico.

\section{- Ley del exceso}

Vivimos en la era de la información y el conocimiento. Probablemente todos coincidiremos al señalar la gran abundancia -y a menudo exceso- de información accesible. Pero unido a ello, debemos reconocer que no siempre se va a transformar en conocimiento y que además se podrán determinar significativas diferencias individuales en la generación de conocimiento. Aún teniendo un mismo cincel, un martillo y un gran bloque de mármol, no todos seríamos capaces 
de esculpir el David de Miguel Ángel. Es por ello que se torna fundamental entender la gestión del conocimiento como una parte fundamental de la competencia digital del ciudadano del siglo $X X I$. Y desde una perspectiva investigadora, cada vez más se reconoce la relevancia de la revisión sistemática frente a las revisiones literarias de información (Moher y Liberati, 2010). Como ejemplo de un trabajo basado en una revisión sistemática véase Durán, Gutiérrez y Prendes (2015).

\section{- Ley de la caducidad}

Otro elemento a tener en cuenta cuando hablamos de difusión de la investigación es la caducidad de la información en el ámbito de la Tecnología Educativa. Aunque es un hecho significativo en relación con el conocimiento en general, en nuestro campo es especialmente visible por su intrínseca relación con el desarrollo de las tecnologías, pues tal y como planteaban los autores de la corriente filosófica CTS -Ciencia, Tecnología y Sociedad-, las tecnologías no pueden analizarse si no es desde una perspectiva sociosistémica que las ponga en relación con factores contextuales y coyunturales que van más allá de la propia tecnología. Y es por ello aún más grave si cabe que en otras disciplinas del ámbito educativo el hecho de que una publicación de una investigación quede confiscada por el sistema de gestión de las revistas durante muchos meses y, una vez aprobado, no se publique hasta mucho después. Las tecnologías digitales y las versiones pre-print vienen en gran medida a ayudarnos a salvar estos escollos de la temporalidad y la caducidad de las investigaciones.

Tras estas metáforas, se puede deducir cuál es nuestra visión de lo que ha sido y debería ser la Tecnología Educativa, que recordemos es el tema de interés de RIITE:

"una disciplina que se encarga del estudio de los medios, en tanto que recursos en procesos formativos, y por ende es desde la Tecnología Educativa desde donde estudiaremos el uso de las TIC en el proceso de enseñanza-aprendizaje (tanto en contextos formales como no formales), su uso e influencia en el aprendizaje informal y así mismo, el impacto de las tecnologías en el mundo educativo en general. Estudiar este impacto de las tecnologías supone analizarlas en relación con los factores sociales, culturales, económicos, políticos... que influyen de modo continuo y constante en su puesta en práctica. Es un enfoque sociosistémico en el cual la Tecnología Educativa analiza procesos mediados y siempre desde una perspectiva holística e integradora" (Serrano et al., 2016, p.11).

Antes de concluir, retomemos el título que encabeza este artículo y recordemos que el sentido de esta nueva revista es apoyar a los jóvenes investigadores en su proceso de difusión de sus trabajos (los márgenes), promover una publicación que por sí misma sea original en cuanto a su planteamiento de las publicaciones, apoyándonos en todo tipo de herramientas digitales y en la propia comunidad científica a modo de red de conocimiento (disrupción) y centrar nuestro foco de interés en la Tecnología Educativa (en busca de ella).

\section{COMIENZA LA RED...}

Por último, no queremos concluir esta presentación sin hacer una breve reseña de los artículos que conforman nuestro primer número.

\section{Dr. Francisco Martínez (Universidad de Murcia): Sentado en el Ánden}

En este artículo se realiza una invitación a la reflexión crítica acerca de la evolución de la Tecnología Educativa y del contexto en el que se debería desarrollar su investigación. Una reflexión que se centra sobre todo en el ámbito de la educación superior, la Universidad, como lugar natural para investigar y difundir lo que supone la incorporación de estas tecnologías en la enseñanza y por extensión en la sociedad. Observar desde el andén, como las TIC han logrado situarse como una especialidad en sí mismas creando un distanciamiento de la Tecnología Educativa, dejando tras de sí un empobrecimiento conceptual y de justificación pedagógica de aquellas dentro de la acción didáctica. 


\section{Dr. Julio Cabero (Universidad de Sevilla): ¿Qué debemos aprender de las pasadas investigaciones en Tecnología Educativa?}

La Tecnología Educativa (TE) ha sido una de las disciplinas de la Ciencias de la Educación que más ha evolucionado en el terreno de las Ciencias de la Educación, aunque también debemos reconocer que en los últimos tiempos se ha dado un cierto retroceso de sus principios, que afortunadamente se está superando, por su equiparación desde ciertos sectores con la aplicación de las Tecnologías de la Información y Comunicación (TIC). En su evolución han intervenido diferentes acontecimientos que van desde las transformaciones ocurridas en las corrientes psicológicas que tradicionalmente la han fundamentado, la significación adquiridas por las TIC en la sociedad del conocimiento con la aparición constantes de tecnologías emergentes, el reclamo de ampliación de su fundamentación teórica, y el asumir que no es algo monolítico y por tanto transferible y aplicable en cualquier contexto. Lógicamente estas transformaciones también han repercutido en las visiones existentes sobre su investigación, encontrándonos con un aumento no solo en su producción y temáticas, sino también, en un cambio de paradigmas, metodologías, perspectivas, preguntas de investigación y agenda de investigación. Para analizar su investigación en el artículo se pretenden responder a dos preguntas: ¿qué debemos aprender de los pasados estudios de investigación en TE? y ¿cómo abordar las futuras investigaciones en TE?

\section{Dr. Xavier Carreras*, Dr. Juan González** y Dr. Jordi Coiduras* (*Universidad de Lleida y **Universidad Rovira y Virgili)}

\section{Ética e investigación en Tecnología Educativa: necesidad, oportunidades y retos}

A pesar de que la ética no ocupa un lugar privilegiado en los estudios del ámbito de la tecnología educativa, su transversalidad nos debe llevar a considerar su importancia en tres grandes ámbitos: la ética como compromiso de acción y de investigación (que parte de la cada vez más expresa recomendación institucional de respeto hacia principios éticos sólidos y compartidos), la ética como regulación del investigador (que le exige el respeto de esos principios generales en el quehacer concreto del proceso de investigación) y la ética como objeto de estudio (que entiende que las diferentes respuestas éticas a las diversas situaciones tiene un interés intrínseco que merece ser estudiado). De todo ello se reflexiona en el presente artículo, a fin de dar pautas que ayuden a promover investigaciones sobre tecnología educativa seguras, éticas y responsables.

\section{Dra. Bárbara de Benito y Dr. Jesús Salinas (Universidad de Islas Baleares) \\ La Investigación Basada en Diseño en Tecnología Educativa}

Se entiende por Investigación Basada en Diseño un tipo de investigación orientado hacia la innovación educativa cuya característica fundamental consiste en la introducción de un elemento nuevo para transformar una situación. Este tipo de investigación trata de responder a problemas detectados en la realidad educativa recurriendo a teorías científicas o modelos disponibles de cara a proponer posibles soluciones a dichos problemas. A este fin, se diseñan programas, paquetes didácticos, materiales, estrategias didácticas, etc.., que se someten a pruebas y validación, y, una vez mejorados, se difunden a la realidad escolar. El proceso de investigación presenta, generalmente, dos etapas: investigar hasta crear un nuevo producto y sus sucesivas mejoras, y por otro lado aportar conocimiento en forma de principios que contribuyen a nuevos procesos de diseño. Entendiendo producto no sólo objetos materiales (libros de texto, programas de vídeo, aplicaciones de ordenador, juegos de simulación,), sino también procesos y procedimientos (métodos de enseñanza, planes de organización escolar, estrategias didácticas, distintos programas...). 


\section{Dr. Jesús Valverde (Universidad de Extremadura):}

La investigación en Tecnología Educativa y las nuevas ecologías del aprendizaje: Design-Based Research (DBR) como enfoque metodológico.

La investigación en Tecnología Educativa ha experimentado un amplio crecimiento en las últimas décadas. Este desarrollo no ha tenido una influencia significativa en la práctica educativa. La integración de las tecnologías digitales en los sistemas educativos no ha modificado sustancialmente la metodología docente para adaptarse a las competencias del siglo XXI. La investigación educativa tiene un escaso impacto en la toma de decisiones de los profesionales de la educación. Por otra parte, las nuevas ecologías del aprendizaje nos sitúan ante contextos de enseñanza-aprendizaje más flexibles, abiertos, distribuidos y conectados. Se necesitan nuevas teorías del conocimiento y nuevos enfoques en las metodologías de investigación educativa. En este artículo el «aprendizaje trialógico» se identifica como un marco conceptual útil para la comprensión de los nuevos contextos educativos y el «Design-Based Research» (DBR) se describe como un modelo metodológico idóneo para la integración efectiva de la investigación en la teoría y práctica pedagógica.

Dra. Mercé Gisbert, Dr. Juan González y Dr. Francesc Esteve (Universidad Rovira i Virgili)

Competencia digital y competencia digital docente: una panorámica sobre el estado de la cuestión

La investigación en Tecnología Educativa ha venido desarrollando en los últimos años los conceptos de competencia digital del estudiante y competencia digital docente. El primero tiene que ver con las capacidades necesarias para aprender en la Sociedad de la Información; el segundo, con la necesidad de ser competente en el uso de la tecnología educativa como docente. En relación con ambos conceptos, la investigación aplicada ha avanzado considerablemente en nuestro contexto. Detallamos los principales hitos hasta la fecha: conceptualizaciones, diseño de instrumentos de diagnóstico, desarrollo de rúbricas de evaluación y exploración de posibilidades $3 \mathrm{D}$ para el desarrollo de las competencias. $\mathrm{Y}$, por último, se abordan las principales líneas de investigación que deben desarrollarse en el futuro de forma preferente.

\section{Dra. Rosabel Roig y Dr. Saulius Rosales-Statkus (Universidad de Alicante)}

\section{El Relato Digital. Análisis de sus elementos y tipología}

El Relato Digital, en su forma más conocida, es un vídeo corto, de aproximadamente dos a tres minutos de duración, en que se combinan imágenes estáticas y/o en movimiento, música, efectos de sonido, etc., con una narración creada y grabada por el autor del vídeo, en el que comunica un mensaje que incluye su punto de vista tratando de involucrar a la audiencia. A pesar de ello, existen distintas visiones de lo que debe ser un Relato Digital, a partir de las cuales se han definido tipos y elementos que éstos deben contener para lograr una comunicación más efectiva del mensaje que portan. En el presente trabajo pretendemos ahondar en estas características que han hecho que el Relato Digital se haya asociado en el ámbito de la educación con aspectos tales como la alfabetización digital, medios de comunicación y aprendizaje colaborativo, entre otros.

\section{Víctor González y Mª del Mar Román (Universidad de Murcia)}

\section{Investigador novel: estrategias y herramientas en el mundo conectado}

El momento en el que un estudiante comienza con el proceso investigador, ya sea en un trabajo fin de master o una tesis, se encuentra en muchas ocasiones desbordado por la gran cantidad de cosas nuevas que debe manejar en poco tiempo. Para este momento existen una serie de herramientas que nos permiten regular todo el proceso, siendo de interés para el propio investigador algunos enfoques como el de Entorno Personal de Aprendizaje. De este 
modo, nos encontramos con herramientas que nos van a permitir realizar una buena búsqueda de información en las bases de datos, herramientas que facilitan la gestión de la abundante información que encontraremos en dicho proceso así como programas estadísticos -tanto cuantitativos como cualitativos- que van a hacer que el proceso de análisis de datos sea algo menos complicado.

\section{REFERENCIAS BIBLIOGRÁFICAS}

Aliaga, F. M. (2014). Veinte años de publicación electrónica y de acceso abierto: la madurez de una pionera. RELIEVE, 20 (1), pp.1-11. DOI: http://10.7203/relieve.20.1.3856

Arnaiz, P. y Prendes M.P. (Coords.) (2010). Tecnologías para los docentes: camino recorrido y mirada hacia el futuro en la Universidad de Murcia. Murcia: Editum.

Costa, C. (2013). The habitus of digital scholars. Research in Learning Technology, 21.

Delgado, E. (2015). Las revistas electrónicas en acceso abierto: pasado, presente y futuro. RELIEVE, 21 (1), pp. 1-15. DOI: 10.7203/relieve.21.1.5005

Durán, M., Gutiérrez, I. y Prendes, M.P. (2015). Definición de un modelo de competencia digital del profesorado universitario: diseño a partir de una revisión sistemática. En Ruiz-Palmero, J., Sánchez-Rodríguez, J. y Sánchez-Rivas, E. (Edit.). Innovaciones con tecnologías emergentes. Málaga: Universidad de Málaga.

Durat, J. M. y Mengual-Andrés, S. (2014). Impacto de la Sociedad del Conocimiento en la universidad y en la comunicación científica. RELIEVE, 20 (2), pp. 1-14. DOI: http://10.7203/relieve.20.2.4343

Kaltura (Ed.) (2015). The State of Video in Education 2015: a Kaltura Report. Nueva York: Kaltura. Recuperado de: http://site.kaltura.com/rs/984-SDM859/images/The_State_of_Video_in_Education_2015_a_Kaltura_Report.pdf

Moher, D. y Liberati, A. (2010). Declaración PRISMA: una propuesta para mejorar la publicación de revisiones sistemáticas y metaanálisis. Medicina Clínica, 135 (11), pp. 505506.

Orduña, E., Martín, A. y Delgado, E. (2016). La bibliometría que viene: almetrics (autor level metrics) y las múltiples caras del impacto de un autor. El profesional de la información, 25 (3), pp. 485-496. DOI: http://dx.doi.org/10.3145/epi.2016.may.18

Prendes, M.P. y Sánchez, M.M. (2014). Arquímedes y la tecnología educativa: un análisis crítico en torno a los MOOC. Revista Interuniversitaria de Formación del Profesorado. 79, 29-49.

Ren, X. (2013). Beyond Open Access: Open Publishing and the Future of Digital Scholarship. 30th Annual conference on Australian Society for Computers in Learning in Tertiary Education, ASCILITE.

Ruiz-Corbella, M., Galán, A. y Diestro, A. (2014). Las revistas científicas de Educación en España: evolución y perspectivas de futuro. RELIEVE, 20 (2), pp. 1-27. DOI: http://10.7203/relieve.20.2.4361

Scanlon, E. (2014). Scholarship in the digital age: Open educational resources, publication and public engagement. British Journal of Educational Technology 45 (1), pp. 12-23.

Segado-Boj, F. (2013). ¿Revistas 2.0? Revistas científicas españolas del área de Comunicación en las redes sociales. Estudios sobre el Mensaje Periodístico. 19, pp. 10071016. 
Serrano, J.L., Gutiérrez, I. y Prendes, M.P. (2016). Internet como recurso para enseñar y aprender. Una aproximación práctica a la tecnología educativa. Sevilla: Eduforma.

Vázquez-Cano, E. (2013). El videoartículo: nuevo formato de divulgación en revistas científicas y su integración en MOOCs. Revista Comunicar, 41, (21), pp. 83-91.

Veletsianos \& Kimmons (2012). Networked Participatory Scholarship: Emergent techno-cultural pressures toward open and digital scholarship in online networks. Computers \& Education, 58, pp. 766-774.

Veletsianos (2013). Open practices and identity: Evidence from researchers and educators' social media participation. British Journal of Educational Technology, 44 (4), pp. 639-651.

Veletsianos, G. (2012). Higher education scholars' participation and practices on twitter. Journal of Computer Assisted Learning, 28 (4), pp. 336-349.

Weller, M. (2011). The digital scholar: how technology is changing academic practice. Basingstoke: Bloomsburry Academic.

Weller, M. (2014). The Battle for open: How openness won and why it doesn't feel like victory. London: Ubiquity Press. DOI: http://dx.doi.org/10.5334/bam

\section{INFORMACIÓN SOBRE LOS AUTORES}

\section{$M^{\text {a Paz Prendes }}$}

Universidad de Murcia

Doctora en Ciencias de la Educación y Profesora de Tecnología Educativa de la Universidad de Murcia, donde además es Directora del Departamento de Didáctica y Organización Escolar y Directora del Grupo de Investigación en Tecnología Educativa. Coordinadora de los programas interuniversitarios de Máster y Doctorado en Tecnología Educativa en la Universidad de Murcia. Secretaria de la Asociación Edutec para el Desarrollo de la Tecnología Educativa. Directora de RIITE, Revista Interuniversitaria de Investigación en Tecnología Educativa.

\section{José Luis Serrano \\ Universidad de Murcia}

Profesor en el Departamento de Didáctica y Organización Escolar de la Universidad de Murcia. Es Doctor en Tecnología Educativa por la Universidad de las Islas Baleares, Licenciado en Pedagogía, Máster en Psicología de la Educación y Técnico Superior en Educación Infantil. Sus principales líneas de investigación son: TIC en las aulas hospitalarias, Entornos Personales de Aprendizaje (PLEs) e Innovación docente en Educación Superior. Trabaja desde el Grupo de Investigación de Tecnología Educativa de la Universidad de Murcia (http://www.um.es/gite/). Director ejecutivo de RIITE, Revista Interuniversitaria de Investigación en Tecnología Educativa.

Web personal: www.jlserranosanchez.es

\section{$(c)$ EY-NC}

Los textos publicados en esta revista están sujetos a una licencia de Reconocimiento 4.0 España de Creative Commons. Puede copiarlos, distribuirlos, comunicarlos públicamente y hacer obras derivadas siempre que reconozca los créditos de las obras (autoría, nombre de la revista, institución editora) de la manera especificada por los autores o por la revista. La licencia completa se puede consultar en:Licencia Creative Commons Atribución-NoComercial 4.0 Internacional. 